\title{
Per una storia italiana Del tempo PRESENTE: GLI APPALTI, LA MAFIA E IL POTERE SUPPLENTE DELLA MAGISTRATURA
}

\section{Francesco Guerra $^{1}$}

\begin{abstract}
Il presente lavoro è un tentativo di ricostruire una delle pagine più nere della storia italiana recente, quella che si riferisce al periodo delle 'stragi di mafia' (1992-1993), proponendo un'interpretazione non convenzionale, in termini di lettura degli eventi e dal punto di vista più propriamente storiografico. In particolare, a partire da una delle ultime indagini coordinate da Giovanni Falcone e Paolo Borsellino, l'autore ha cercato di mostrare, soprattutto alla data della morte dei due magistrati, il collegamento tra sistema di gestione degli appalti, mafia e, entro una cornice prospettica, potere 'supplente' della magistratura.
\end{abstract}

PAROLE CHIAVE: appalti; mafia; magistratura; Falcone; Borsellino; storia; tempo.

RESUMO: O presente trabalho é uma tentativa de reconstruir uma das páginas mais obscuras da história italiana recente, a que se refere ao período dos 'massacres da máfia' (1992-1993), propondo uma interpretação não convencional, em termos de leitura dos eventos e do ponto de vista mais historiográfico. O autor procurou demonstrar, especialmente a partir de uma das últimas investigações coordenadas por Giovanni Falcone e Paolo Borsellino e, sobretudo pela data da morte dos dois magistrados, a conexão entre sistema de gestão dos contratos públicos, da máfia e, dentro da perspectiva de uma moldura, do poder 'suplente' da magistratura.

PALAVRAS-CHAVE: licitações; máfia; magistratura; Falcone; Borsellino; storia; tempo

ABSTRACT: The present work is an attempt to reconstruct one of the blackest pages of recent Italian history, the one referring to the period of the 'massacres of Mafia' (1992-1993), proposing an unconventional interpretation in terms of the reading of events and from the more properly historiographical point of view. Notably, starting from one of the last investigations directed by Giovanni Falcone and Paolo Borsellino, the author tried to show, especially at the date of the death of the two magistrates, 
the connection between tenders management system, Mafia and, within a perspective context, substitute power of the judiciary.

KEYWORDS: tenders; mafia; judiciary; Falcone; Borsellino; history; time. 
errà, forse, un giorno, che, quando si guarderà retrospettivamente agli eventi accaduti tra la seconda metà del 1992 e l'estate del 1993, si accetterà, senza più infingimenti, il movente che ha portato alla morte di Giovanni Falcone e Paolo Borsellino a così stretta distanza l'uno dall'altro: l'indagine su Mafia e Appalti, avviata da Falcone e condotta dai carabinieri del ROS. In tal modo, si potrà finalmente prendere le distanze da ricostruzioni alternative, quali la presunta 'trattativa Stato-mafia', riconoscendola null'altro che come uno dei tentativi di depistaggio - essendo, gli altri, i primi due processi sulla Strage di Via D' Amelio - messi in atto da parti importanti delle istituzioni italiane, magistratura e alcuni organi di polizia in primis.

Depistaggi, che, peraltro, si scontrano con acclarate risultanze processuali, quale quella consegnata alla sentenza della Corte d'Assise di Catania del 22 aprile 2006, dove, a proposito del movente della strage di via D'Amelio, si afferma "la possibilità che il dottor Borsellino venisse ad assumere la Direzione Nazionale Antimafia e, soprattutto, la pericolosità delle indagini che egli avrebbe potuto svolgere in materia di mafia e appalti" (Sentenza della Corte di Assise di Catania N. 24/06 del 22 Aprile 2006 -12 Settembre 2007).

Risalendo il corso degli eventi di quei terribili anni, occorre dire che, ben prima che la Procura di Milano avviasse l'inchiesta che passerà alla storia come Mani Pulite, nei primi mesi del 1992, già il 20 febbraio 1991 il ROS depositò l'informativa Mafia e Appalti, relativa alla prima parte delle indagini sulle connessioni tra politici, imprenditori e mafiosi, dove si rivelava l'esistenza di un comitato d'affari illegale e si facevano i nomi di società e persone coinvolte.

Come ha scritto in un suo eccellente articolo Luciano Tirinnanzi: "il deposito di Mafia e Appalti fu voluto espressamente da Giovanni Falcone, il quale, all'epoca, stava passando dalla Procura di Palermo alla Direzione degli Affari Penali del Dipartimento di Giustizia di Roma”. Pur di fronte alle titubanze mostrate dal generale Mario Mori, all'epoca a capo del ROS, essendo l'informativa in una fase ancora gestatoria, Falcone si raccomandò con i carabinieri di depositare subito quelle carte, poiché ritenute cruciali per spiegare le connessioni tra mafia, politica e mondo imprenditoriale. "Lo ricordò lo stesso giudice al convegno palermitano del 14 e 15 marzo 1992, a Castello Utveggio, a Palermo: "la mafia è entrata in borsa" - disse due mesi prima di essere ucciso nella Strage di Capaci" (TIRINNANZI, 12 luglio 2013).

Una frase, questa, che, in realtà, stando ad Attilio Bolzoni e al suo libro Faq Mafia, il giudice avrebbe pronunciato già nel 1990 con riferimento alla Calcestruzzi di Raoul Gardini e ai suoi affari, recentemente avviati, con Cosa Nostra (BOLZONI, 2010). A questo proposito va 
parimenti ricordato che Enrico Deaglio retrodata la frase al 1989, collocandola in connessione col fallito attentato dell' Addaura del 20 giugno di quello stesso anno, che avrebbe coinvolto anche i magistrati Carla Del Ponte e Claudio Lehman in visita a Palermo per parlare di un gigantesco riciclaggio di denaro frutto del traffico di stupefacenti nel quale era coinvolto, in maniera pesante, anche l'industriale bresciano Oliviero Tognoli (DEAGLIO, 2010, p. 228).

Pertanto, alla luce di queste diverse testimonianze, appare legittimo postulare che Giovanni Falcone usò più di una volta l'espressione "la mafia è entrata in borsa" negli ultimi anni della sua vita, ciò che può valere come sostanziale elemento di prova della centralità che un simile concetto aveva nella mente del magistrato palermitano. Ma torniamo al dossier su Mafia e appalti del ROS e al 1991.

Quelle carte erano talmente importanti, ricorda Tirinnanzi, che divennero da subito motivo di imbarazzo e indecisione da parte della Procura di Palermo, probabilmente, anche per il diretto coinvolgimento, nel dossier del ROS, di persone vicine a taluni magistrati di quella Procura. Procura, che, non a caso, inizialmente, sulla base del Mafia e Appalti emise solo 5 provvedimenti di custodia cautelare per associazione a delinquere di stampo mafioso (7 luglio 1991), diversamente dai 44 che suggeriva l'informativa. Ma non vi fu solo questo:

\footnotetext{
"Agli avvocati difensori dei 5 arrestati fu indebitamente e insolitamente consegnata l'intera informativa del ROS (890 pagine più 67 di appendice, dunque comprensiva di tutte le 44 persone oggetto d'indagine), anziché stralci dei soli passaggi relativi alle loro posizioni. Con ciò fu svelata l'architettura investigativa complessiva, emersero i nomi di tutti i soggetti nel mirino del ROS e si vanificò l'intera indagine. Dopodiché inizia a scorrere il sangue" (TIRINNANZI, 2013).
}

Il primo a morire fu il deputato andreottiano Salvo Lima, il 12 marzo 1992, l'uomo "delle cosche", come, in modo inappropriato, lo definirono i giornali. Ancora, il 4 aprile del $1992 \mathrm{fu}$ ucciso il maresciallo Giuliano Guazzelli, perché - su esplicita richiesta - rifiutò di stemperare le accuse contro Angelo Siino, Ministro dei Lavori pubblici di Cosa Nostra dopo l'arresto di Vito Ciancimino, nonché uno dei 5 arrestati di Mafia e Appalti e ritenuto dal ROS l'anello di congiunzione tra mafia e imprenditoria. Ancora, il 23 maggio morì Giovanni Falcone e il 19 luglio 1992 la stessa sorte toccò a Paolo Borsellino.

Il 25 giugno del 1992 Paolo Borsellino volle incontrare, segretamente, in una caserma del ROS gli autori dell'informativa, il colonnello Mario Mori e il capitano Giuseppe De Donno, riferendo loro che l'inchiesta Mafia e Appalti era il salto di qualità investigativo che avrebbe permesso di individuare sia i responsabili del gigantesco giro di corruzione siciliana, sia gli autori della morte di Falcone. Borsellino indicò proprio in Mafia e Appalti la causa della morte dell'amico giudice e chiese il massimo riserbo sull'incontro, in particolare nei confronti dei 
colleghi della Procura di Palermo, per timore di fughe di notizie, ma, più ancora, forse, perché non si fidava di alcuni colleghi².

A proposito di Paolo Borsellino è qui necessario fare una piccola digressione, tuttavia sempre legata all'inchiesta su Mafia e Appalti. Quella stessa sera del 25 giugno Borsellino, invitato a parlare al dibattito organizzato dalla rivista Micromega presso l'atrio della Biblioteca Comunale di Palermo, ricevette da Gioacchino Basile, operaio e rappresentante sindacale della Fincantieri di Palermo, un dossier - mai più ritrovato - riguardante i rapporti tra Fincantieri (e le altre ditte che in passato l'avevano preceduta nella gestione dei cantieri palermitani, tra cui la Piaggio) ed esponenti di primo piano di Cosa Nostra, in particolare la cosca dei Galatolo.

Stando a quanto riferitomi da Basile, Borsellino si mostrò molto interessato a quel dossier, chiedendogli di lasciargliene copia quella sera stessa e di inviarne un'altra, a lui indirizzata, presso la Procura di Palermo. In sostanza, all'interno del dossier Basile accusava Fincantieri di appaltare a Cosa Nostra il "lavoro sporco", in particolare lo smaltimento illegale dei rifiuti tossici e quello dell'amianto.

Qui il racconto di Basile si fa ancora più inquietante, chiamando direttamente in causa la Procura di Palermo e i colleghi di Borsellino all'epoca, in particolare uno, varie volte citato da Basile all'interno delle proprie denunce e il cui nome può essere facilmente reperito in rete. Secondo Basile, una delle cause - invero, per lui, la causa - della morte di Paolo Borsellino fu l'interesse mostrato dal magistrato palermitano per questa inchiesta su Fincantieri, che il collega, all'epoca dominus dell'inchiesta, non avrebbe avuto intenzione di svolgere. (BASILE, 27/05/2017)

Entro quest'ottica, le pressioni di Borsellino sul collega lasciano immaginare che, se non fosse intervenuta la strage di via D'Amelio, la suddetta inchiesta su Fincantieri si sarebbe dovuta svolgere. Inchiesta, peraltro, che Borsellino stesso avrebbe potuto coordinare (o quantomeno seguire molto da vicino); ciò che rende plausibile l'ipotesi per cui il magistrato palermitano potesse averla intesa, sin da subito, come una costola della più ampia inchiesta, avviata da Falcone e da lui ereditata, su Mafia e Appalti.

Appare lecito domandarsi, giunti a questo punto, quanto l'interesse mostrato da Borsellino in riferimento a Mafia e Appalti e alla sua ipotizzata "appendice", Fincantieri, da un lato, e dall'altro, le fortissime divergenze tra lui ed elementi di primo piano della Procura di Palermo non siano tracimati dall'ambiente giudiziario sino ad informare Cosa Nostra, venendosi, in tal modo, a configurare, di fatto, come cause, o quantomeno concause, della accelerazione dell'attentato al magistrato, la cui tempistica sembra rimandare a qualcosa di organizzato con estrema rapidità, in particolare considerando che Falcone era stato ucciso nemmeno due mesi prima e in modo tanto eclatante.

Riporto questa tesi così come me l'ha raccontata lo stesso Basile, prestandomi, quindi, ad una storia di tipo congetturale, parimenti, mi preme fare una breve considerazione: questa lettura degli eventi, insieme a Mafia e Appalti, alla quale ritengo sia strettamente collegata, appaiono come i soli possibili moventi dove, per usare le parole del blogger Enrico Tagliaferro, "il cui prodest del reato coincide con i nomi degli autori, ossia le famiglie mafiose coinvolte"

2 Su questo argomento, rimando alle dichiarazioni fatte dal pentito Angelo Siino, in sede processuale, al Sostituto Procuratore Luca Tescaroli e contenute nel libro Perché fu ucciso Giovanni Falcone dello stesso Tescaroli. Cfr. TESCAROLI, 2001, pp. 34-43. 
(Graviano, Madonia e, pur in maniera diversa, Galatolo) (TAGLIAFERRO, 05/06/2017). Per intenderci: sembra qui presente l'interesse diretto delle parti in causa.

Inoltre, bloccare l'inchiesta sembrava essere una soluzione assai difficile da realizzare, da un lato, per l'interesse mostrato da Borsellino ad indagare con forza in quella direzione, dall'altro, perché, sempre Borsellino, era in contatto diretto con Basile. A compendio delle parole di Basile, possiamo rilevare come, dopo la morte di Borsellino, la presente inchiesta non fu realizzata e il dossier consegnato da Basile al magistrato mai più ritrovato, come riferito dalle parole del figlio di Borsellino, Manfredi, allo stesso Basile.

Pertanto, cosa rappresentavano l'informativa del ROS su Mafia e appalti e il corposo dossier sui rapporti tra Fincantieri e Cosa Nostra consegnato da Gioacchino Basile a Paolo Borsellino? La mia idea è che gli elementi qui richiamati rappresentino, ove legati fra loro, il momento centrale di coagulazione dei differenti interessi di cui erano espressione alcuni segmenti, marci, della società palermitana, siciliana e italiana del periodo (dalla mafia all'imprenditoria, dalla politica a una certa magistratura ideologizzata) con significativi addentellati di natura finanziaria - in particolare banche e varie e complesse figure di intermediari (Oliviero Tognoli, ad esempio) dediti per lo più a riciclare e reinvestire il denaro sporco - fuori dalla Sicilia. In questo senso non stupisce che chi si oppose a questa trasversale convergenza di interessi, quali ad esempio Giovanni Falcone e Paolo Borsellino, sia stato spazzato via.

A supporto della tesi qui presentata giova succintamente ricordare la cronologia relativa all'archiviazione dell'inchiesta su Mafia e Appalti: il 13 luglio 1992 viene depositata, da parte dei Sostituti Procuratori Guido Lo Forte e Roberto Scarpinato, la richiesta di archiviazione dell'inchiesta; il 22 luglio il Procuratore Capo, Pietro Giammanco, controfirma la suddetta richiesta di archiviazione, che verrà, infine, eseguita il 14 agosto 1992.

A onor del vero, va anche detto che il 19 luglio, di buon mattino, alle 7:00, Giammanco chiamò Borsellino a casa, affidandogli la delega sulle indagini riguardanti gli appalti. Un fatto, questo, che, a giudicare dai pessimi rapporti che intercorrevano tra i due magistrati, potrebbe apparire insolito e che, proprio per questo, solleva un interrogativo di non poco conto, come fattomi notare dal giornalista Giampiero Casagni: e se Giammanco, al netto delle iniziali resistenze, si fosse davvero persuaso che vi fossero elementi della Procura di Palermo decisi ad archiviare il più rapidamente possibile l'inchiesta su Mafia e Appalti - tesi, questa, più che fondatamente attribuibite a Borsellino - e avesse, perciò, chiamato il collega per informarlo che la richiesta di archiviazione, solo pochi giorni prima, il 13 luglio, era giunta sulla sua scrivania?

Sia come sia, vi è che, anche in seguito all'archiviazione dell'inchiesta, la frattura all'interno della Procura di Palermo tra alcuni magistrati e il ROS, in particolare le figure apicali dell'epoca, sembra non essersi mai più sanata. Un plastico esempio di questo clima furono le denunce del colonnello Giuseppe De Donno nel processo a carico del generale Mario Mori e del colonnello Mauro Obinu, accusati di favoreggiamento aggravato a Cosa Nostra per la mancata cattura del boss Bernardo Provenzano; processo conclusosi con l'assoluzione dei due imputati. "L'informativa su Mafia e Appalti, condotta agli inizi degli anni '90 dai Carabinieri del ROS di Palermo dopo la consegna alla Procura nel febbraio del '91 - dichiarò De Donno - 
venne congelata fino al giugno di quell'anno".

Continua De Donno: "I risultati dell'informativa su cui avevamo lavorato per molto tempo insieme con il giudice Falcone furono estremamente ridotti. Da gennaio a giugno non ci fu alcun riscontro dell'attività svolta. A giugno poi la Procura chiese e ottenne cinque ordinanze di custodia cautelare, ma ritengo che la portata dall'inchiesta fu sottovalutata. Noi eseguimmo gli arresti, ma nacque un contenzioso con la Procura che non accettò una serie di nostre indicazioni" (TIRINNANZI, 2013).

A questo punto, preme svolgere una nuova, breve digressione, necessaria per comprendere più a fondo il complesso sistema che faceva da base alla relazione tra mafia e appalti, spostandoci dal piano della ricostruzione storica a quello più specificamente socio-giuridico, al fine di constatare come, diversamente da ciò che succede nel campo dell'edilizia urbana e di piccole e medie opere, nel quale le imprese mafiose possono avere un ruolo preminente, alle gare relative ai grandi appalti tali imprese concorrono molto di rado. A sconsigliare la loro partecipazione è l'elevata visibilità pubblica dell'affare, come pure le particolari competenze necessarie alla realizzazione dei lavori, delle quali sono in genere sprovviste. Ad esempio, per la costruzione di grandi opere infrastrutturali nel Mezzogiorno l'interlocutore dell'ente appaltante è, di norma, una grande e specializzata impresa del Centro-Nord, spesso con un profilo internazionale. Una volta aggiudicatosi l'appalto, per realizzare concretamente i lavori, la grande impresa li suddivide in subappalti, affidandoli in molti casi, -documentati da indagini giudiziarie, - a imprese locali "consigliate" dai gruppi criminali.

Tali imprese locali possono avere caratteristiche anche molto diverse tra loro, in termini di capacità tecniche e gestionali, ed essere più o meno strettamente legate ai gruppi criminali. Entro questo contesto il Mafia e Appalti viene a rappresentare uno snodo fondamentale anche per il coinvolgimento, in questa informativa, di alcune cooperative operanti sul territorio siciliano e più in generale su quello italiano.

A questo proposito vale la pena richiamare il saggio di Ferdinando Imposimato, Giuseppe Pisauro e Sandro Provvisionato, Corruzione ad Alta Velocità. Viaggio nel governo invisibile, come pure quello di Francesco Bigazzi e Valentin Stepankov, Il viaggio di Falcone a Mosca, con la preziosa prefazione scritta dal giudice Carlo Nordio. La tesi che unisce questi due lavori è che, a partire dal dossier su Mafia e appalti, Falcone avrebbe ampliato le sue indagini sulle "cooperative rosse" (vale a dire legate all'ex Partito Comunista Italiano) fino ad arrivare, da un lato, a scoprire il gigantesco giro di tangenti che si trovava alla base della costruzione della linea ad alta velocità su varie tratte ferroviarie italiane, mentre, dall'altro, seguendo il flusso del denaro - "seguire i piccioli" era il suo specifico tratto investigativo - sarebbe risalito all'ingente traffico di soldi che dalla Russia affluiva in Italia e qui, con ogni probabilità, tramite le cooperative, veniva reinvestito illegalmente nelle tangenti e nei cantieri legati all'alta velocità e, in Sicilia non meno che in altre regioni d'Italia, in appalti attraverso un sodalizio tra alcune cooperative e Cosa Nostra.

Purtroppo, tali indagini, a causa della prematura scomparsa di Falcone e Borsellino, non sono mai potute approdare a esiti definitivi, ciò anche per il salvifico e tempestivo intervento 
della famosa inchiesta Mani pulite, la quale, a partire dal febbraio 1992, ha eliminato dalla scena politica italiana i due maggiori partiti della cosiddetta Prima Repubblica: la Democrazia Cristiana e il Partito Socialista Italiano; tuttavia salvando, non casualmente, il Partito Comunista dalle grandi inchieste, che pure l'avrebbero visto coinvolto.

Approssimandomi alla conclusione di questo saggio, sento di dovere esprimere il mio personale, ma ancorché fondato, scetticismo riguardo alla possibilità di comprendere, in maniera approfondita, ciò che nel 1992 e negli anni a seguire accadde in Sicilia ${ }^{3}$ senza legarlo a quel vero colpo di Stato (definizione, questa, contenuta nel libro The Italian Guillotine: Operation Clean Hands and the Overthrow of Italy's First Republic di Stanton H. Burnett e Luca Mantovani), che fu l'operazione Mani pulite condotta a partire dal febbraio 1992 dalla Procura di Milano.

In sede di ricerca storiografica, ciononostante, è possibile, almeno, tentare di riscrivere il perimetro generale degli eventi e delle causalità. Compito, questo, per il quale mi avvarrò di alcune puntuali considerazioni svolte dall'avvocato e columnist de La voce di New York, Fabio Cammalleri, da anni studioso, avvertito e meticoloso, delle più recenti vicende della storia italiana.

Pertanto: "non sono le stragi del biennio 1992/1993 che impongono la ricerca di dimensioni criminali più approfondite, ma è un pregiudizio ideologico interno alla magistratura italiana, tesa a trasformarla in potere supplente, che si avvale delle stragi per consolidarsi;

Questo pregiudizio ideologico o, se si preferisce, questa trasformazione della magistratura in vero e proprio potere supplente dello Stato, non nasce a Palermo, ma a Palermo trova la sua "occasione storica" di decisiva manifestazione e affermazione;

Nasce tra Roma e Milano, a partire dal Congresso di Magistratura Democratica, una delle associazioni di magistrati in cui si articola l'Associazione Nazionale Magistrati, del 1970, e sviluppa la sua prima fase fino alla cattura di Mario Moretti, uno dei capi storici delle Brigate Rosse; poi comincia il Maxiprocesso contro la Nuova Camorra Organizzata di Raffaele Cutolo ed è, questo, un primo reimpiego su larga scala della delazione fuori del terreno terroristico e, contemporaneamente, una prima estensione del potere giudiziario oltre i propri confini, appunto trasformandolo in un potere supplente rispetto agli altri due poteri (esecutivo e legislativo), oltre Magistratura Democratica;

A questo punto, però, questo potere supplente viene spiazzato dal lavoro del Pool Antimafia - di cui facevano parte Falcone e Borsellino - che usa quegli stessi strumenti giudiziari (uso molto attento dei pentiti per esempio), ma in modo non ideologico (ossia non per i fini del potere supplente). È questo il momento nel quale il potere supplente entra in fibrillazione;

La fibrillazione è risolta in favore dell'ideologia (del potere supplente) per il tramite

3 Faccio qui riferimento, in maniera non esaustiva, all'omicidio del politico democristiano Salvo Lima, alla Strage di Capaci e a quella di via D'Amelio, fino alla stagione dei 'processi politici' a Palermo sotto la direzione della Procura da parte di Giancarlo Caselli (Corrado Carnevale, Giulio Andreotti, Calogero Mannino, Bruno Contrada, fino a Marcello Dell'Utri e ai vari tentativi di coinvolgere nella fattispecie di concorso esterno in associazione mafiosa Silvio Berlusconi). 
di Tangentopoli, in particolare dell'inchiesta Mani Pulite, sebbene sia preceduta da alcuni eventi specifici, quali il fallito attentato dell'Addaura a Falcone (21 giugno 1989). L'ideologia, a questo punto, si è ormai fatta carattere costitutivo generale di un Apparato di potere nato a vita nuova;

Le stragi hanno matrice esclusivamente in Cosa Nostra, ma i suoi cadaveri sono fatti oggetto di cattura ideologica, che permette l'ulteriore fase dell'assestamento dell'originaria pregiudiziale ideologica propria del potere supplente incarnato dalla magistratura;

L'intelligence (qui il riferimento è al processo e alla condanna di Bruno Contrada) vince su Cosa Nostra e, inizialmente lasciata lavorare, viene poi liquidata: perché avrebbe potuto costituire un altro fattore di fibrillazione, in quanto, come Falcone, conosceva la stessa grammatica usata dal potere supplente, ma agiva in modo non ideologico;

Infine, dopo l'arresto del boss mafioso Pietro Aglieri (6 giugno 1997), comincia l'eliminazione, già avviata con Bruno Contrada, di tutti coloro che potevano rappresentare un ostacolo all'affermazione di tale potere supplente; è la fase del consolidamento, gli anni di ciò che in precedenza si è definito col termine 'processi politici'. Questa ultima fase è quella della 'eliminazione in assetto stabilizzato"”.

Più nel dettaglio, quale era il fulcro di questi ‘processi politici'? Tali processi si basavano sul cosiddetto principio - peraltro esplicitamente riconosciuto dalla Corte di Cassazione della 'convergenza del molteplice'. Solo che un simile principio non rappresenta uno strumento infallibile. Appare di tutta evidenza, come ha osservato Salvo Giorgio, riferendosi al calvario giudiziario di Bruno Contrada, che "la 'convergenza del molteplice' non può essere un'arbitraria estensione del principio secondo cui quae singula non probant simul unita (ciò che non viene dimostrato da una singola prova, viene dimostrato da più prove insieme). Ciò perché è vero che, in generale, più prove concordanti possono formare un convincimento, ma devono essere prove certe e non indizi. Questo anche alla luce del fatto che si potrebbe arrivare alla paradossale conclusione secondo cui due o più prove inattendibili (o non con certezza attendibili, e quindi non valutabili), se considerate congiuntamente, potrebbero giustificare la condanna di un individuo, laddove la valutazione globale delle prove mostrerebbe l'inattendibilità delle singole prove» (GIORGIO, 2008) ${ }^{4}$. Motivo per cui sarebbe preferibile, in luogo di questo problematico principio di 'convergenza del molteplice', basare le singole indagini sul ben più attendibile elemento del 'riscontro esterno' o 'oggettivo'.

Purtroppo, soprattutto alla luce di quanto scritto con riferimento al potere supplente esercitato dalla magistratura nei vari 'processi politici', brevemente richiamati all'interno di questo saggio, non solo il principio del 'riscontro esterno' non poteva essere contemplato, mancandone le condizioni sotto il profilo delle più basilari norme del diritto, ma, più ancora, non doveva, in nessun modo e in nessun momento, essere contemplato.

4 Ad esempio: se dico che $\mathrm{X}$ ha rubato una certa quantità di denaro, o si ritrova una parte di questo denaro, perlomeno il suo percorso, per usare un'immagine cara a Giovanni Falcone, oppure, in sede processuale, questa accusa contro $\mathrm{X}$ viene a cadere. 


\section{Riferimenti bibliografici}

BASILE, G. Conversazione privata: 27/05/2017.

BIGAZZI, F., STEPANKOV, V. Il viaggio di Falcone a Mosca. indagine su un mistero italiano. Milano: Mondadori, 2015, pp. 156.

BOLZONI, A. Faq mafia. Milano: Bompiani, 2010.

https://books.google.com.br/books?id=-qKgDQAAQBAJ\&pg=PT68\&dq=la+mafia+è+entrata+in+ borsa+Bolzoni\&hl=it\&sa=X\&ved=0ahUKEwiY2rKliLHbAhVDiZAKHfI1 A1cQ6wEIKTAA\#v=onepa ge \&q=la\%20mafia\%20è\%20entrata\%20in\%20borsa\%20Bolzoni\&f=false. Accesso: 10/06/2017.

BURNETT, S.H., MANTOVANI, L., The Italian Guillotine: Operation Clean Hands and the Overthrow of Italy's First Republic. Lanham: Rowman \& Littlefield, 1998, p. 331.

CAMMALLERI, F., Conversazione privata: 15/06/2017.

DEAGLIO, E. Patria 1978-2010. Milano: il Saggiatore, 2010, p. 228.

GIORGIO, S. La verità su Bruno Contrada. http://casocontrada.blogspot.com/2008/06/ sommario_1696.html. Accesso: 20/06/2017.

IMPOSIMATO, F., PISAURO, G., PROVVISIONATO, S. Corruzione ad Alta Velocità. Viaggio nel governo invisibile. Roma: Koinè Nuove Edizioni, 1999, p. 192.

TAGLIAFERRO, E., Conversazione privata: 05/06/2017.

TESCAROLI, L., Perché fu ucciso Giovanni Falcone. Soveria Mannelli: Rubbettino, 2001, p. 34-43.

TIRINNANZI, L. Mafia e Appalti, una verità scomoda, in Panorama, 12 luglio 2013.

Recebido: 06/08/2019

Aprovado: 31/10/2019 